\title{
Quadratic Optimal Regulator Design of a Pneumatic Control Valve
}

\author{
Mohammad Heidari ${ }^{1}$ and Hadi Homaei ${ }^{2}$ \\ ${ }^{1}$ Mechanical Engineering Group, Islamic Azad University, Aligudarz Branch, P.O. Box 159, Aligudarz, Iran \\ ${ }^{2}$ Faculty of Engineering, Shahrekord University, P.O. Box 115, Shahrekord, Iran \\ Correspondence should be addressed to Mohammad Heidari; moh104337@yahoo.com
}

Received 30 May 2013; Revised 20 September 2013; Accepted 4 October 2013

Academic Editor: Abdelali El Aroudi

Copyright (C) 2013 M. Heidari and H. Homaei. This is an open access article distributed under the Creative Commons Attribution License, which permits unrestricted use, distribution, and reproduction in any medium, provided the original work is properly cited.

Pneumatic control valves are still the most used devices in the process industries, due to their low cost and simplicity. This paper presents a regulator for pneumatic control valves using pole-placement method, optimal control, full-order state observer, and minimum-order state observer and their responses will be compared with each other. Bondgraph method has been used to model the control valve. Simulation results have been made for four models of regulator. The results show that minimum overshoot and settling time are achieved using optimal regulator of pneumatic valve.

\section{Introduction}

Process plants consist of hundreds, or even thousands, of control loops all networked together to produce a product to be offered for sale. Each of these control loops is designed to keep some important process variables such as pressure, flow, level, and temperature. within a required operating range to ensure the quality of the end product. Each of these loops receives and internally creates disturbances that detrimentally affect the process variable, and interaction from other loops in the network provides disturbances that influence the process variable. To reduce the effect of these load disturbances, sensors and transmitters collect information about the process variable and its relationship to some desired set point. A controller then processes this information and decides what must be done to get the process variable back to where it should be after a load disturbance occurs. When all the measuring, comparing, and calculating are done, some type of final control element must implement the strategy selected by the controller. The most common final control element in the process control industries is the control valve. The control valve manipulates a flowing fluid, such as gas, steam, water, or chemical compounds, to compensate for the load disturbance and keep the regulated process variable as close as possible to the desired set point. Control valves adjust the temperature, pressure, flow rate, and so forth by changing the flow rate. Figure 1 shows a sliding-stem pneumatic control valve. Pneumatic control valves are still the most used valves in the process industries, due to their low cost and simplicity. Pneumatic valves are used extensively in various industries today. Industry standard has been established that details the vibration, humidity, thermal, salt spray, and temperature extremes that these valves must operate within. This makes the design of valve control systems a very challenging task. Control valves have two major components, valve body housing and the actuation unit. One factor in the quality of the final end product is the improvement of the control loop performance. A critical component in the loop is the final control element, the control valve package. Optimized actuator parameters play a vital role in the dynamic performance of the pneumatic control valve. Champagne and Boyle [1] reviewed the pneumatic actuator and positioner parameters that affect the control package performance. This is done through the use of a control valve package computer model to assess the dynamic performance. The attributes of spring return versus double acting actuators are illustrated. The effects of supply pressure, step size, load margin, flow, actuator volume, and design style are investigated through the use of mathematical simulations of pneumatic control valve dynamic performance. Hagglund presented a procedure that 


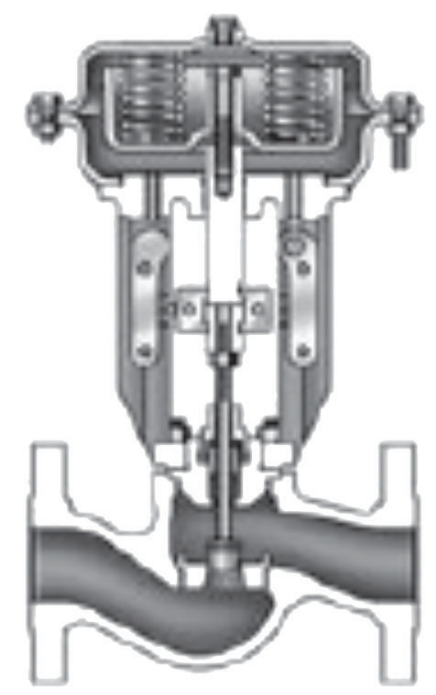

FIgURE 1: Pneumatic control valve with diaphragm.

compensates for static friction (stiction) in pneumatic control valves [2]. The compensation is obtained by adding pulses to the control signal. The characteristics of the pulses are determined from the control action.

The compensator is implemented in industrial controllers and control systems, and the industrial experiences show that the procedure reduces the control error during stickslip motion significantly compared to standard control without stiction compensation. The oscillations caused by static friction (stiction) in pneumatic control valves cause losses in quality and expense of raw materials. The input-output behavior of a pneumatic control valve affected by stiction in valve. De Souza et al. [3] presented a well-known stiction compensation method that reduces variability both at process variable and pneumatic valve stem movement. The two-move method is revisited in this paper and it was shown that assumptions on the knowledge of steady-state stem position of control valve that assures equality of set point and the controlled variable were not easily achievable. Bondgraph is a graphical representation of a physical dynamics system. It is similar to the better known block diagram and signal flow, with the major difference that the arcs in bondgraphs represent bidirectional exchange of physical energy, while those in block diagrams and signal-flow graphs represent unidirectional flow of information. Also, bondgraphs are multidomain and domain neutral. This means that a bondgraph can incorporate multiple domains simultaneously. Bondgraphs were devised by Paynter [4] at MIT in April 1959 and subsequently developed into a methodology together with Karnopp et al. [5]. Early prominent promoters of bondgraph modeling techniques among others were Thoma [6], Dixhoorn, and Dransfield. Athanasatos and Costopoulos [7] used the bondgraph method for finding the proactive fault in $4 / 3$ way direction control valve of a high pressure hydraulic system. The accuracy of the bondgraph model was verified by comparing its response to the response of an actual hydraulic system. Díaz-Zuccarini et al. [8] utilized the bondgraph as boundary condition for a detailed model of an idealized

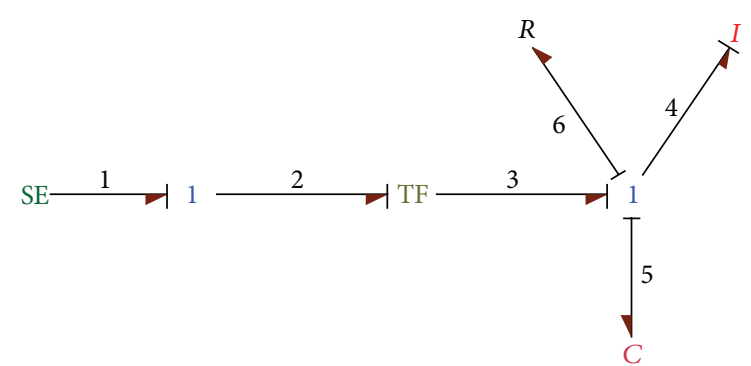

FIGURE 2: Bondgraph control valve actuator.

mitral valve. A specific application in cardiovascular modeling was demonstrated by focusing on a specific example: a 3D model of the mitral valve coupled to a lumped parameter model of the left ventricle.

In this study a pneumatic control valve is modeled by bondgraph method. A regulator has been designed using pole-placement method, optimum regulator, full-order state observer, and minimum-order state observer for this system. In continuing, their responses have been compared with each other.

This research is organized as follows. Section 2 recalls the bondgraph model of valve and proposes equations of motion of valve. Section 3 develops quadratic optimal regulator of pneumatic valve. Sections 4 and 5 present designing a regulator by pole-placement technique; full and minimum order observers, respectively. Section 6 shows the results of full and minimum order regulator observers and quadratic optimal regulator of valve and finally some conclusions are given in Section 7.

\section{Bondgraph Model of Valve and Transfer Function}

The bondgraph model of the valve is shown in Figure 2. In this model, SE is the inlet pressure of the system. The pressure changes to force by multiplying in effect area of the diaphragm. In bondgraph, this transformer is modeled by T.F. Element $R$ is the friction of the system. Element $I$ is the movable mass of valve and diaphragm.

Element $C$ represents the spring of the valve actuator. Also 1-junction is a common flow junction. 1-junctions have equality of flows and the efforts sum up to zero with the same power orientation. In fact, junctions can connect two or more bonds. The direction of the half arrows $(\rightarrow)$ denotes the direction of power flow given by the product of the effort and flow variables associated with the power bond. The bonds in a bondgraph may be numbered sequentially using integers starting with 1 . The two 1-junctions in the bondgraph shown can be uniquely identified as (S 12 ) and (S 45 6); similarly, symbols like $S E_{1}$ and $R_{6}$ can be used to identify a particular element. This system has two state variables $P_{4}$ and $q_{5} \cdot q_{5}$ is the displacement of valve stem and the variation of the spring length. Also $v_{4}=P_{4} / I_{4}$ is the velocity of the valve stem. The 
equations of motion are derived using bondgraph method as follows:

$$
\begin{gathered}
\dot{P}_{4}=A \times S E_{1}-K_{3} q_{5}-\frac{R_{6}}{I_{4}} P_{4}, \\
\dot{q}_{5}=\frac{P_{4}}{I_{4}} .
\end{gathered}
$$

Now, if the velocity and position of stem are zero in the initial condition, $X(0)$, then we have

$$
X(0)=\left[\begin{array}{l}
P_{4}(0) \\
q_{5}(0)
\end{array}\right]=\left[\begin{array}{l}
0 \\
0
\end{array}\right] .
$$

By derivation of relation (2) with respect to time, we have

$$
\ddot{q}_{5}=\frac{\dot{P}_{4}}{I_{4}} .
$$

By substitution of $\dot{P}_{4}$ from (1) into (4), we have

$$
\ddot{q}_{5}=\frac{A \times S E_{1}-K_{3} q_{5}-\left(R_{6} / I_{4}\right) P_{4}}{I_{4}} .
$$

By substitution of $P_{4}$ from (2) into (5), we have

$$
\ddot{q}_{5}=\frac{1}{I_{4}}\left(A \times S E_{1}-K_{3} q_{5}-R_{6} \dot{q}_{5}\right) .
$$

Using laplace transformation of (6), we have

$$
\frac{q_{5}(s)}{S E_{1}(s)}=\frac{\left(A / I_{4}\right)}{s^{2}+\left(R_{6} / I_{4}\right) s+\left(K_{3} / I_{4}\right)} .
$$

Equation (7) is the transfer function of the valve. The results of bondgargh model of valve show that the response of the system is identical with the result in $[9,10]$.

\section{Quadratic Optimal Regulator of Pneumatic Valve}

Let us consider the system that is defined as form of a statespace representation as follows:

$$
\begin{aligned}
& \dot{x}=A x+B u, \\
& y=C x+D u,
\end{aligned}
$$

where $x$ is called the state vector, $\dot{x}$ the derivative of the state vector with respect to time, $y$ the output vector, and $u(t)$ the input or control vector. Also $A$ is the system matrix, $B$ the input matrix, $C$ the output matrix, and $D$ direct transmission matrix. We will now consider the optimal control problem that, given the system (8), the matrix $K$ should be determined, so that

$$
u(t)=-K x(t)
$$

It means that the control signal is determined by the state of that moment. The dimension of the state feedback gain matrix is $1 \times n$ and $n$ is the number of the states. This minimizes the performance index $(J)$ as

$$
J=\int_{0}^{\infty}\left(x^{T} Q x+u^{T} R u\right) d t
$$

where $Q$ is a positive-definite (or positive semidefinite) Hermitian or real symmetric matrix and $R$ is a positivedefinite Hermitian or real symmetric matrix [11].

Note that the second term on the right side of (11) accounts for the expenditure of the energy of the signal control. In this problem, we assume that the control vector $u(t)$ is unconstrained. The linear control law given by (11) is the optimal control law. Therefore, if the unknown elements of the matrix $K$ are determined so as to minimize the performance index, then $u(t)=-K x(t)$ is optimal for any initial state $x(0)$. Hence,

$$
K=R^{-1} B^{T} P .
$$

Equation (12) gives the optimal matrix. Thus the optimal control law to the quadratic optimal control problem where the performance index is given by (11) is linear and given by

$$
u(t)=-K x(t)=-R^{-1} B^{T} P x(t) .
$$

The matrix $P$ in (12) must satisfy the following reduced equation:

$$
A^{T} P+P A-P B R^{-1} B^{T} P+Q=0 .
$$

Equation (14) is called the Riccati reduced-matrix equation.

\section{Designing a Regulator by Pole-Placement Technique}

Suppose that the system is defined by (8) and the control signal is given by (10). The feed back gain matrix $\mathrm{K}$ that forces the eigenvalues of $A-B K$ to be $\mu_{1}$ and $\mu_{2}$ (desired values) can be determined by the following steps. Firstly, the controllability of the system is checked. The controllability matrix is defined as follows:

$$
M=\left[\begin{array}{ll}
A & A B
\end{array}\right] .
$$

For this system, the rank of the controllability matrix is two. In the next step, the characteristic polynomial of matrix $A$,

$$
|s I-A|=s^{2}+a_{1} s+a_{2},
$$

determines the values of $a_{1}$ and $a_{2}$. Now the desired closedloop poles $\mu_{1}$ and $\mu_{2}$ are determined based on the transient response or frequency response requirements, such as speed, damping ratio, and steady state requirements. The desired characteristic equation becomes

$$
\left(s-\mu_{1}\right)\left(s-\mu_{2}\right)=s^{2}+\alpha_{1} s+\alpha_{2} .
$$

The values of $\alpha_{1}$ and $\alpha_{2}$ are determined from (17). The required state feedback gain matrix $K$ can be determined from (18):

$$
K=\left[\begin{array}{ll}
\alpha_{2}-a_{2} & \alpha_{1}-a_{1}
\end{array}\right] T^{-1},
$$


where

$$
T=M W
$$

Matrix $W$ is given by

$$
W=\left[\begin{array}{cc}
a_{1} & 1 \\
1 & 0
\end{array}\right] .
$$

\section{Full- and Minimum-Order State Observer}

If not all state variables are available for feedback, unavailable state variables should be estimated by observer. Suppose that observer observes all state variables of the system, regardless of whether some state variables are available for direct measurement. For this situation we must use full-order state observer and observe all state variables. State observers can be designed if and only if the observability condition is satisfied. Necessary and sufficient condition for observability condition is that the dual of the original system must be completely state controllable [9].

The complete state observability for this system is that the rank of

$$
N=\left[\begin{array}{ll}
C^{T} & A^{T} C^{T}
\end{array}\right]
$$

is 2 because the system is a two-degree-of-freedom system. Characteristic equation can be obtained from (16). We should determine two groups of desired poles as each one has two poles. The first group relates to system state variables and the other one relates to observer variables. Therefore, state observer gain matrix can be obtained for both situations. We choose two dominant poles based on the transient response requirements and remained poles assignment so enough far from two dominant poles. State observer gain matrix can be determined from (19)-(23). Also matrix $W$ can be obtained by using (16)-(20) after specifying observer desired poles. Subsequently, we have

$$
K_{e}=\left(W N^{T}\right)^{-1}\left[\begin{array}{ll}
\alpha_{2}-a_{2} & \alpha_{1}-a_{1}
\end{array}\right]^{T} .
$$

The transfer function of the full-order controller observer is

$$
\frac{U(s)}{-Y(s)}=K\left(s I-A+K_{e} C+B K\right)^{-1} K_{e} .
$$

For designing of minimum-order state observer, we should traverse the following stages. By selecting eigenvalues for observer matrix, the characteristic equation is

$$
\left|s-A_{b b}+K_{e} A_{a b}\right|=s+\widehat{\alpha}_{1},
$$

where $\widehat{\alpha}_{1}$ is the desired eigenvalue for the minimum-order observer. The minimum observer gain $K_{e}$ can be determined by choosing the desired eigenvalue for the minimum-order observer and then the procedure developed for the full-order observer with appropriate modifications. We have

$$
A=\left[\begin{array}{ll}
A_{a a} & A_{a b} \\
A_{b a} & A_{b b}
\end{array}\right], \quad B=\left[\begin{array}{l}
B_{a} \\
B_{b}
\end{array}\right] .
$$

The minimum observer gain is defined as follows:

$$
K_{e}=\left(\widehat{W} \widehat{N}^{T}\right)^{-1}\left(\widehat{\alpha}_{1}-\widehat{a}_{1}\right),
$$

where

$$
\widehat{N}=A_{a b}, \quad \widehat{W}=\widehat{a}_{1} .
$$

Note that $\widehat{a}_{1}$ is the coefficient in the characteristic equation for the state equation

$$
\left|s-A_{b b}\right|=s+\widehat{a}_{1} .
$$

The transfer function for minimum-order state observer is given by the following formula:

$$
\frac{U(s)}{-Y(s)}=-\left[\widetilde{C}(s-\widetilde{A})^{-1} \widetilde{B}+\widetilde{D}\right],
$$

where

$$
\begin{gathered}
\widetilde{A}=\widehat{A}-\widehat{F} K_{b}, \\
\widetilde{B}=\widehat{B}-\widehat{F}\left(K_{a}+K_{b} K_{e}\right), \\
\widetilde{C}=-K_{b}, \\
\widetilde{D}=-\left(K_{a}+K_{b} K_{e}\right),
\end{gathered}
$$

where

$$
\begin{gathered}
\widehat{A}=A_{b b}-K_{e} A_{a b}, \\
\widehat{B}=\widehat{A} K_{e}+A_{b a}-K_{e} A_{a a}, \\
\widehat{F}=B_{b}-K_{e} B_{a} .
\end{gathered}
$$

\section{Results and Discussion}

Table 1 shows the parameters of a sliding-stem pneumatic control valve.

By substitution of Table 1 into (7), we have

$$
\frac{q_{5}(s)}{S E_{1}(s)}=\frac{6.53}{s^{2}+33.33 s+226333.3} .
$$

Matrices of the state space equations of the valve are as follows:

$$
\begin{gathered}
A=\left[\begin{array}{cc}
0 & 1 \\
-226333.33 & -33.33
\end{array}\right], \\
B=\left[\begin{array}{c}
0 \\
914.2
\end{array}\right], \quad C=\left[\begin{array}{ll}
1 & 0
\end{array}\right], \quad D=0 .
\end{gathered}
$$

In this section, we consider the design of regulator system with full- and minimum-order observers and then quadratic optimal regulator of pneumatic valve. The detail of designing a full and minimum order observer is given in the previous section. Let us design a regulator which achieves a $10 \%$ maximum overshoot and settle time less than 0.5 second for output when the initial condition is $x(0)=\left[\begin{array}{ll}0 & 0.1\end{array}\right]^{T}$. 
TABLE 1: Valve parameters [9].

\begin{tabular}{lcc}
\hline Name of variable & Parameter & Value \\
\hline Effective area of diaphragm & $A$ & $0.196 \mathrm{ft}^{2}$ \\
Spring constant & $K$ & 6790 \\
Movable mass & $I(M)$ & $0.03 \mathrm{slug}$ \\
Resistance and friction & $R$ & $1 \mathrm{lb} \cdot \mathrm{S} / \mathrm{ft}$ \\
coefficient & $\mathrm{SE}$ & $140 \mathrm{lb} / \mathrm{ft}^{2}$ \\
Air pressure & &
\end{tabular}

A $10 \%$ overshoot and a settling time of 0.5 second yield $\xi=0.591$ and $\omega_{n}=13.53$; thus, the characteristic equation for dominant poles is $s^{2}+16 s+183.1=0$, where the dominant poles are located at $-8 \pm j 10.91$ [12]. Hence, choose the desired closed-loop poles at $s=\mu_{i}(i=1,2)$, where

$$
\mu_{1}=-8+j 10.91, \quad \mu_{2}=-8-j 10.91 .
$$

In the pole placement method, we must first check the rank of the controllability matrix from (15):

$$
M=\left[\begin{array}{cc}
0 & 914.2 \\
914.2 & -30470
\end{array}\right] .
$$

Since the rank of matrix $M$ is 2 , arbitrary pole placement is possible. In this case for determining the state feedback gain matrix $K=\left[\begin{array}{ll}k_{1} & k_{2}\end{array}\right]$, the characteristic polynomial for the desired system is

$$
\begin{aligned}
\mid s I & -A+B K \mid \\
& =\left|\left[\begin{array}{ll}
s & 0 \\
0 & s
\end{array}\right]-\left[\begin{array}{cc}
0 & 1 \\
-226333.33 & -33.33
\end{array}\right]+\left[\begin{array}{c}
0 \\
914.2
\end{array}\right]\left[\begin{array}{ll}
k_{1} & k_{2}
\end{array}\right]\right| \\
& =s^{2}+\left(914.2 k_{2}+33.33\right) s+914.2 k_{1}+2.2633 e 5 .
\end{aligned}
$$

The characteristic polynomial must be equal to

$$
\begin{aligned}
\left(s+\mu_{1}\right)\left(s+\mu_{2}\right) & =(s+8+j 10.91)(s+8-j 10.91) \\
& =s^{2}+16 s+183.1 .
\end{aligned}
$$

By equating the coefficients of the terms of the like powers of $s$, we obtain

$$
k_{1}=-247.34, \quad k_{2}=-0.019,
$$

or

$$
K=\left[\begin{array}{ll}
-247.34 & -0.019
\end{array}\right] .
$$

Response to initial condition with pole placement method is shown in Figure 3. Note that the maximum overshoot of valve is about $0.4 \%$ for output, and settling time is about 0.5 second. The design is thus acceptable.

The input, $u(t)=-k x(t)$, can be calculated from the previously calculated matrices, $k$ and $x$. The control input, $u(t)$, which is a force is applied to the valve stem. The larger the control input magnitude, the bigger the energy spent by

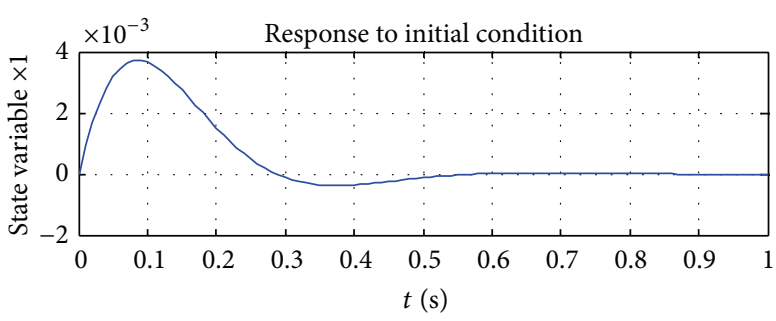

(a)

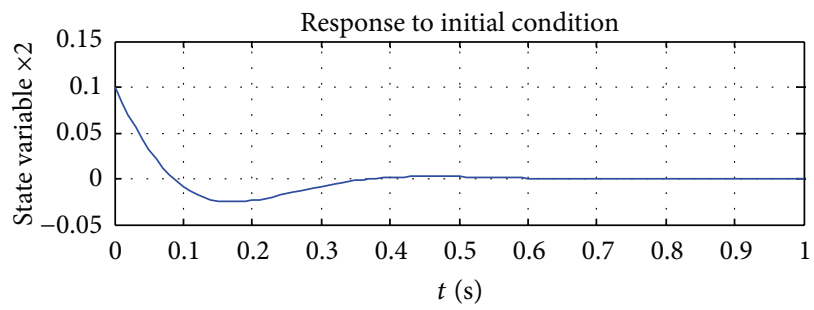

(b)

Figure 3: Response to initial condition.

the actuator in generating the control input, and the higher the cost of control. As shown in Figure 6, the maximum value of input control signal is about 0.9 . Observability matrix can be obtained from (21) as

$$
N=\left[\begin{array}{ll}
1 & 0 \\
0 & 1
\end{array}\right]
$$

Since the rank of observability matrix is 2 , the given system is completely state observable. Hence, full-order observer is applicable. Assume that the desired eigenvalues for full-order observer are $\lambda_{1}=-116$ and $\lambda_{2}=-116$. The state feedback gain matrix $K$ for this case is obtained as follows:

$$
K=\left[\begin{array}{ll}
-247.3751 & -0.019
\end{array}\right] \text {. }
$$

The full observer gain matrix $K_{e}$ is calculated as follows:

$$
K_{e}=\left[\begin{array}{ll}
200 & -219500
\end{array}\right]^{T} .
$$

The transfer function of the full-order observer controller $\left(G_{s f}\right)$ is obtained as follows:

$$
G_{s f}(s)=\frac{-331800 s-52710000}{s^{2}+1343 s+213300} .
$$

Figure 4 shows results of the initial response of stem valve with full-order observer controller. Note that the maximum overshoot of valve is about $0.15 \%$ for output, and settling time is 0.5 second. The design is thus acceptable. In this case, the maximum value of input control signal is about 0.4 .

The minimum order observer is of first order. Assume that the desired eigenvalue for minimum order observer is $\lambda_{1}=-40$.

The state feedback gain matrix $K$ is obtained as

$$
K=\left[\begin{array}{ll}
-247.3751 & -0.019
\end{array}\right] .
$$


TABLE 2: Settling time, overshoot, and maximum control input of the response to initial condition of the valve.

\begin{tabular}{lccc}
\hline Method for regulator design & Settling time $(\mathrm{sec})$ & Overshoot (\%) & Maximum control input \\
\hline Pole placement & 0.5 & 0.4 & 0.9 \\
Full-order observer & 0.5 & 0.15 & 0.4 \\
Minimum-order observer & 0.5 & 0.25 & 0.6 \\
Optimal regulator & 0.1 & 0.018 & 0.005 \\
\hline
\end{tabular}

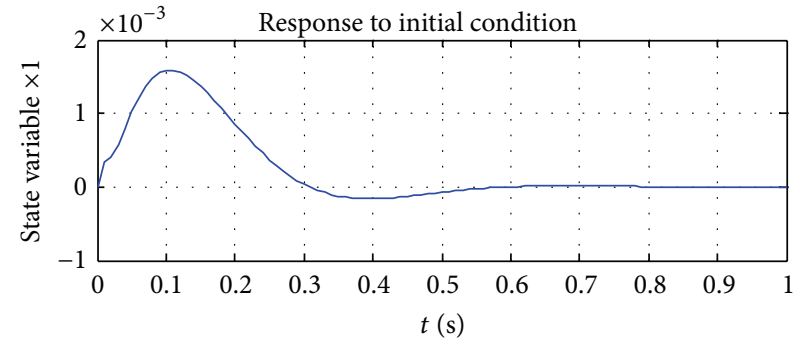

(a)

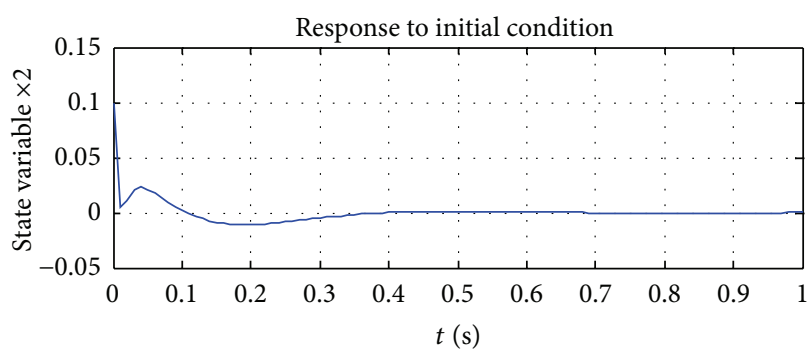

(b)

FIGURE 4: Response to initial condition with full-order observer.

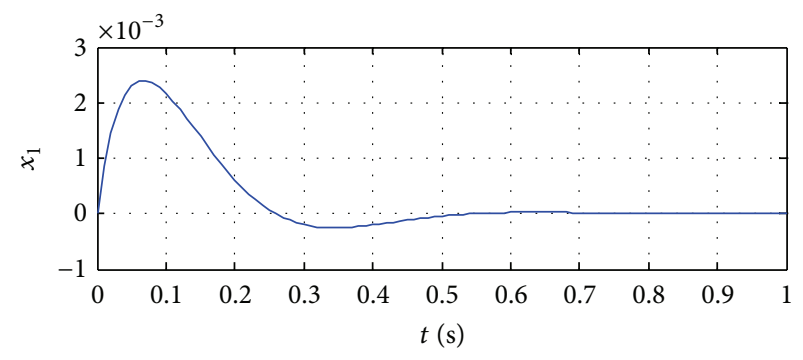

(a)

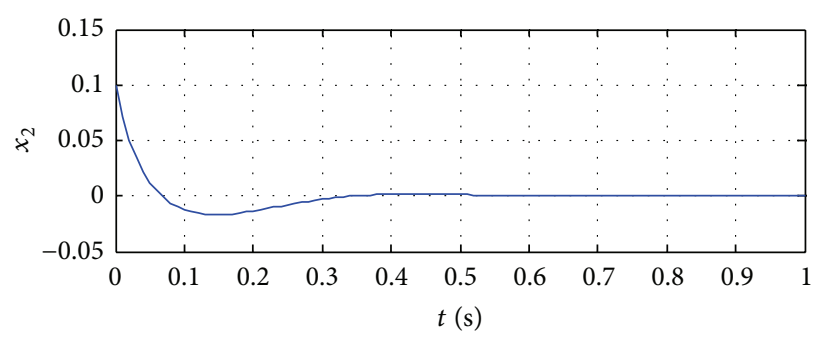

(b)

FIGURE 5: Response of system to initial condition with minimum-order observer.

And the minimum observer gain $K_{e}$ can be obtained as

$$
K_{e}=6.67 \text {. }
$$

The transfer function of the minimum-order observer controller $\left(G_{s m}\right)$ is obtained as follows:

$$
G_{s m}(s)=\frac{-248.3 s-15500}{s+62.67} .
$$

Figure 5 shows results of the initial response of valve stem with minimum-order observer controller. The initial condition is assumed as $x(0)=\left[\begin{array}{ll}0 & 0.1\end{array}\right]^{T}$. Note from Figure 5 that the maximum overshoot of valve is about $0.25 \%$ for output, and settling time is about 0.5 second. The design is thus acceptable. In this case, control input, $u(t)$, is shown in Figure 6, and the maximum value is about 0.6.

Figure 6 shows the comparison of control input signal of the valve with pole placement method (pole), full-order observer (full), and minimum-order observer (mini).

We will now consider the optimal control problem of valve. For this purpose we choose by trial and error $R=1$ and $Q=\left[\begin{array}{cc}0.01 & 0 \\ 0 & 0.01\end{array}\right]$. Solving (14) for $P$, we have

$$
P=\left[\begin{array}{cc}
39.45 & 21 e-6 \\
21 e-6 & 15 e-5
\end{array}\right] .
$$

Substituting $P$ into (12), the optimum gain matrix will be as follows:

$$
K=\left[\begin{array}{ll}
0 & 0.07
\end{array}\right]
$$

The initial condition is assumed as $x(0)=\left[\begin{array}{ll}0 & 0.1\end{array}\right]^{T}$. The results of the overshoot and settling time to step input for closed loop system are shown in Figure 7. As shown in Figure 7, the overshoot is very small and less than $0.018 \%$. Also the settling time is $0.1 \mathrm{sec}$.

The input, $u(t)=-k x(t)$, can be calculated from the previously calculated matrices, $k$ and $x$. The control input for the value of the optimal regulator gain matrix is shown in Figure 8. Note that the maximum input value is about 0.005 .

Transient response parameters are given in Table 2 for comparing four methods of regulator design with each other. All designing of regulators of the valve are under the same initial condition.

\section{Conclusion}

The aim of this study was the development of design some regulators to meet transient response specifications of a pneumatic control valve. The mathematical model of 


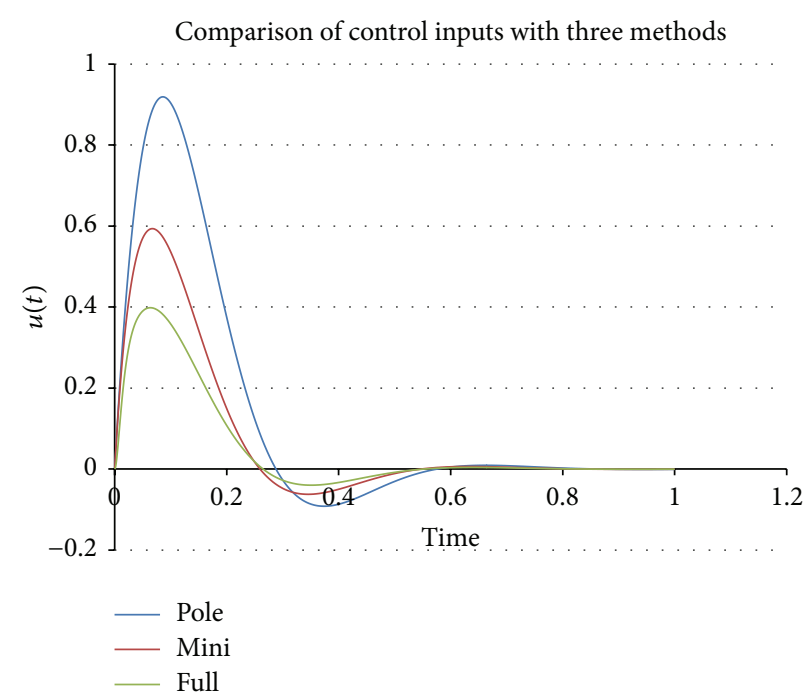

FIgURE 6: The control input, $u(t)$, of the system with three methods.

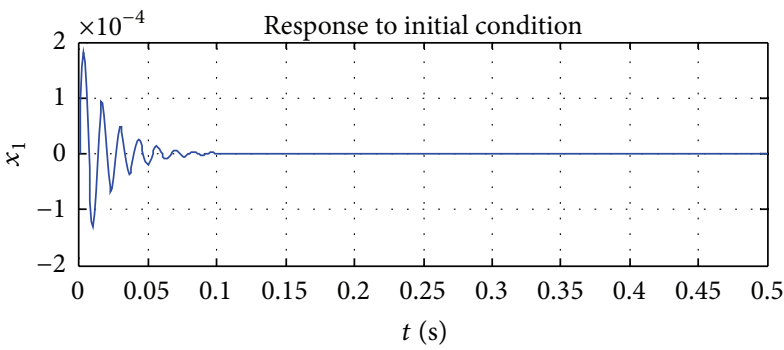

(a)

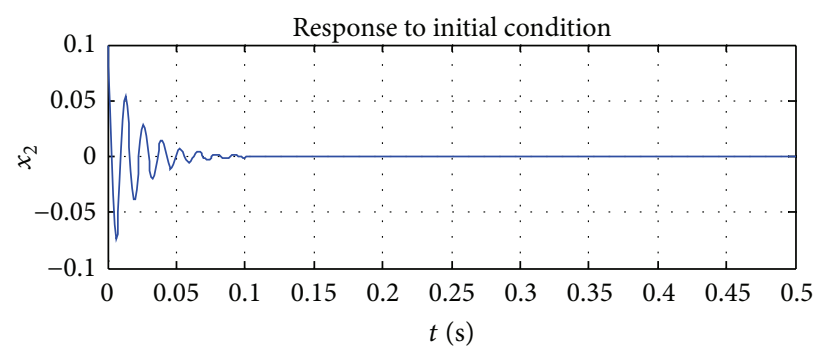

(b)

FIGURE 7: Closed-loop initial response of valve for optimal regulator designed with $Q$ and $R=1$.

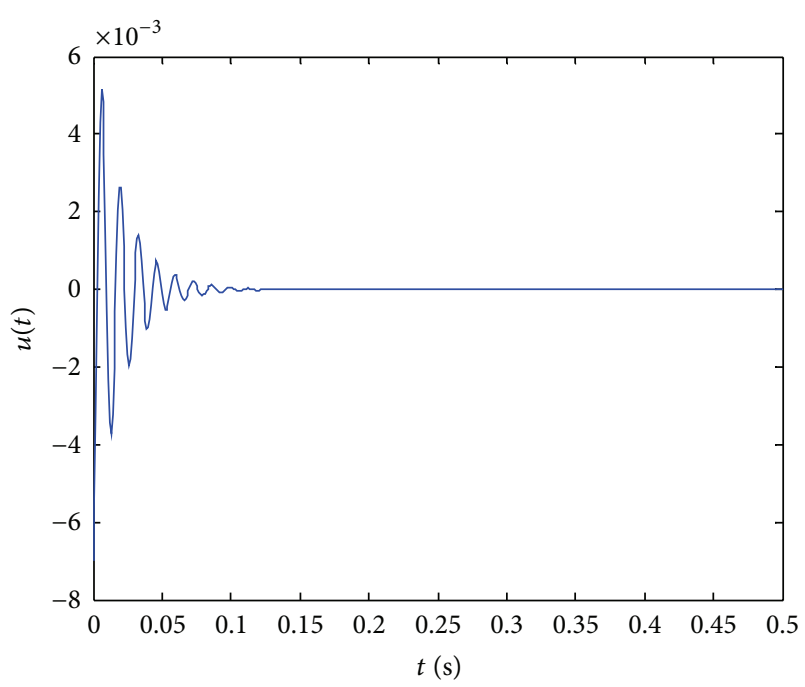

FIgURE 8: The control input $u(t)$ of the system (with quadratic optimal).

system and subsequently state equations were derived using bondgraph method. Regulator has been designed using pole-placement method, optimal control, full-order state observer and minimum-order state observer for this system. Also, their responses and then important transient response parameters were derived and a comparison between the four designed regulators was made. It was observed that responses of the pole-placement technique and minimumorder observer are near to each other. The full order observer regulator has the most high overshoot percentage among the other regulators. The results show that the minimum overshoot and settling time are achieved in quadratic optimal regulator of pneumatic valve.

\section{References}

[1] R. P. Champagne and S. J. Boyle, "Optimizing valve actuator parameters to enhance control valve performance," ISA Transactions, vol. 35, no. 3, pp. 217-223, 1996.

[2] T. Hägglund, "A friction compensator for pneumatic control valves," Journal of Process Control, vol. 12, no. 8, pp. 897-904, 2002.

[3] M. A. de Souza, L. Cuadros, C. J. Munaro, and S. Munareto, "Improved stiction compensation in pneumatic control valves," Computers and Chemical Engineering, vol. 38, pp. 106-114, 2012.

[4] H. Paynter, Analysis and Design of Engineering Systems, M.I.T. Press, 1959. 
[5] D. C. Karnopp, R. C. Rosenberg, and D. L. Margolis, System Dynamics: Modeling, Simulation, and Control of Mechatronic Systems, John Wiley \& Sons, 5 edition, 2012.

[6] J. U. Thoma, Simulation By Bondgraphs: Introduction To A Graphical Method, Springer, 2012.

[7] P. Athanasatos and T. Costopoulos, "Proactive fault finding in a 4/3-way direction control valve of a high pressure hydraulic system using the bond graph method with digital simulation," Mechanism and Machine Theory, vol. 50, pp. 64-89, 2012.

[8] V. Díaz-Zuccarini, D. Rafirou, J. LeFevre, D. R. Hose, and P. V. Lawford, "Systemic modelling and computational physiology: the application of Bond Graph boundary conditions for 3D cardiovascular models," Simulation Modelling Practice and Theory, vol. 17, no. 1, pp. 125-136, 2009.

[9] J. C. Mackanic, Design, Construction and Evaluation of $A$ Simulated Geothermal Flow System, University of California, 1980.

[10] M. Heidari and H. Homaei, "Stem control of a sliding-stem pneumatic control valve using a recurrent neural network," Advances in Artificial Neural Systems, vol. 2013, Article ID 410870, 7 pages, 2013.

[11] K. Ogata, Modern Control Engineering, Prentice Hall, 5 edition, 2010.

[12] N. S. Nise, Control System Engineering, John Wiley \& Sons, 6 edition, 2010. 

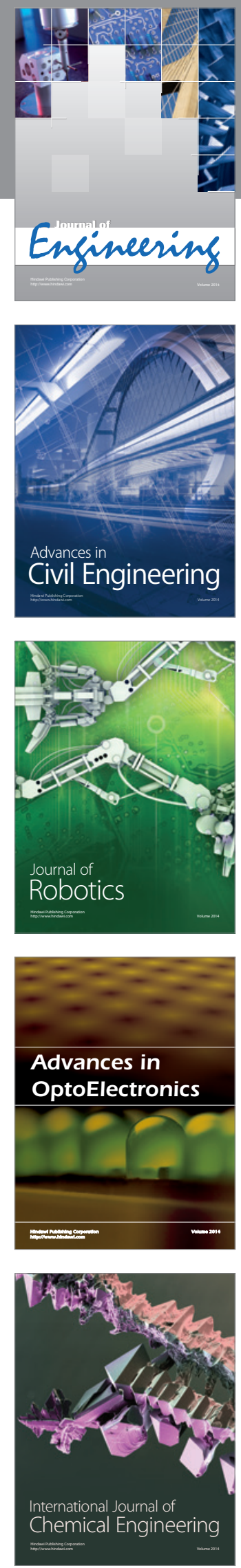

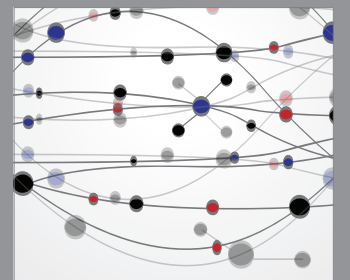

The Scientific World Journal
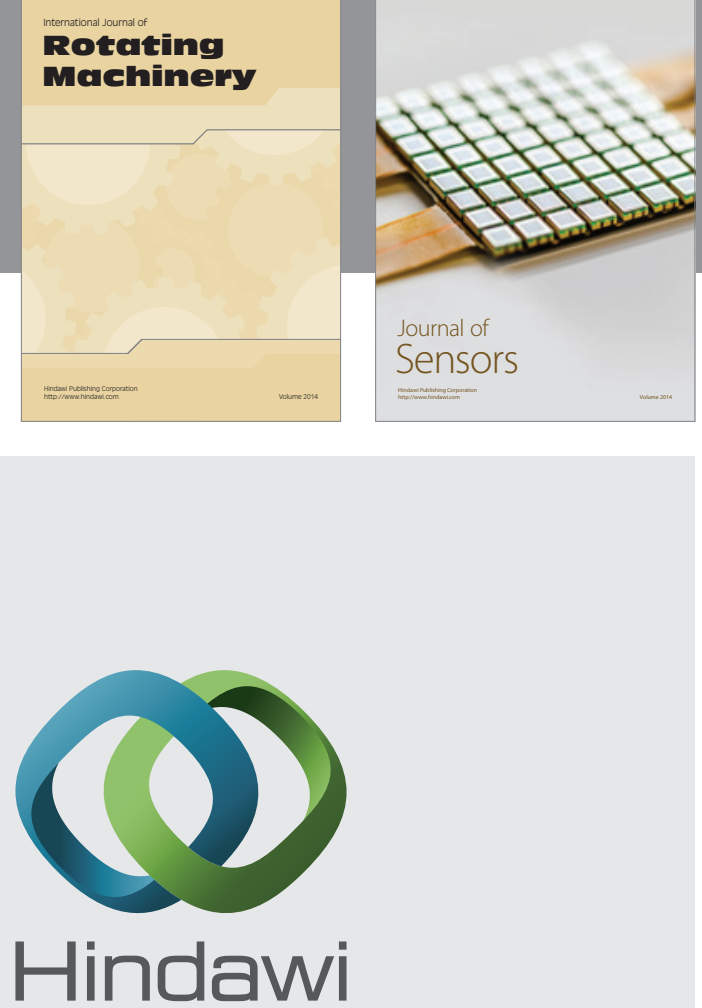

Submit your manuscripts at http://www.hindawi.com
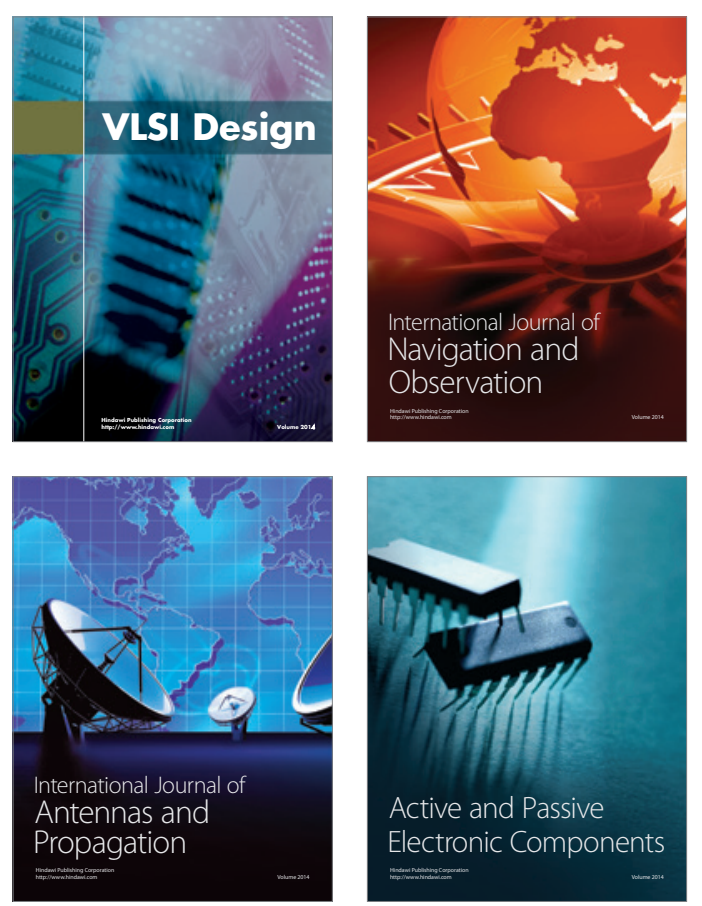
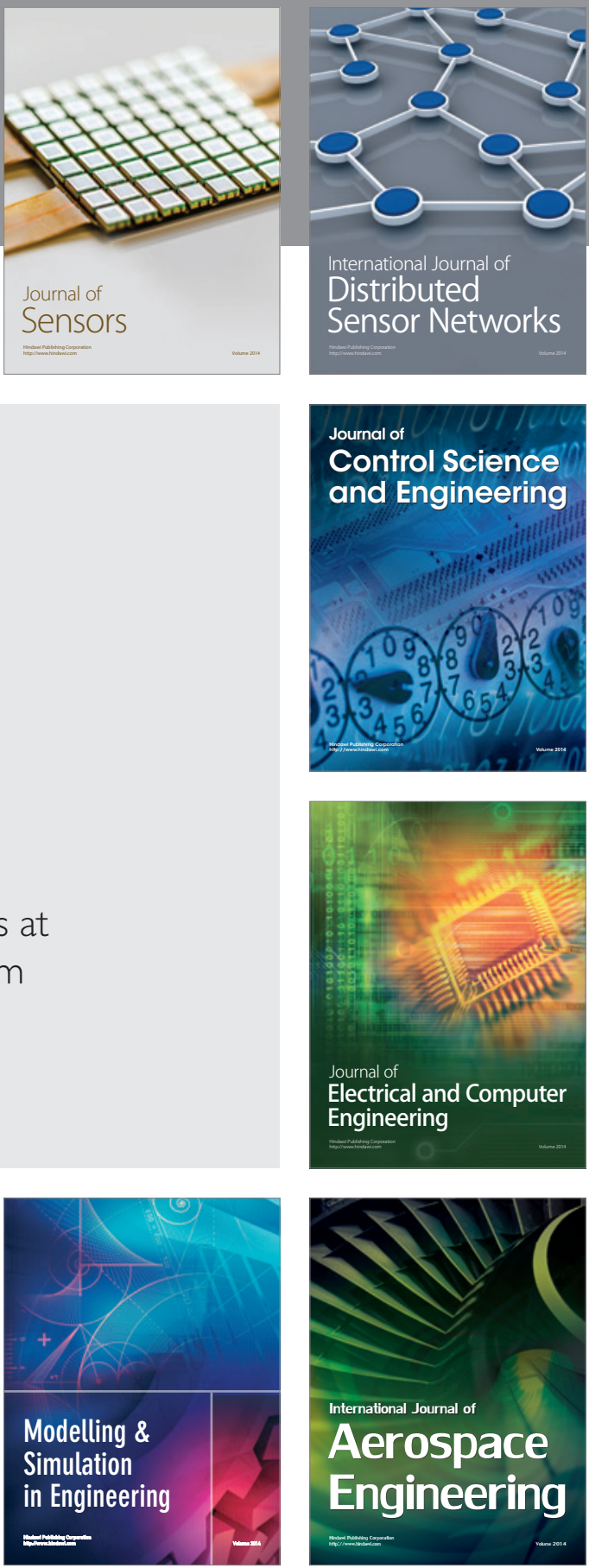

Journal of

Control Science

and Engineering
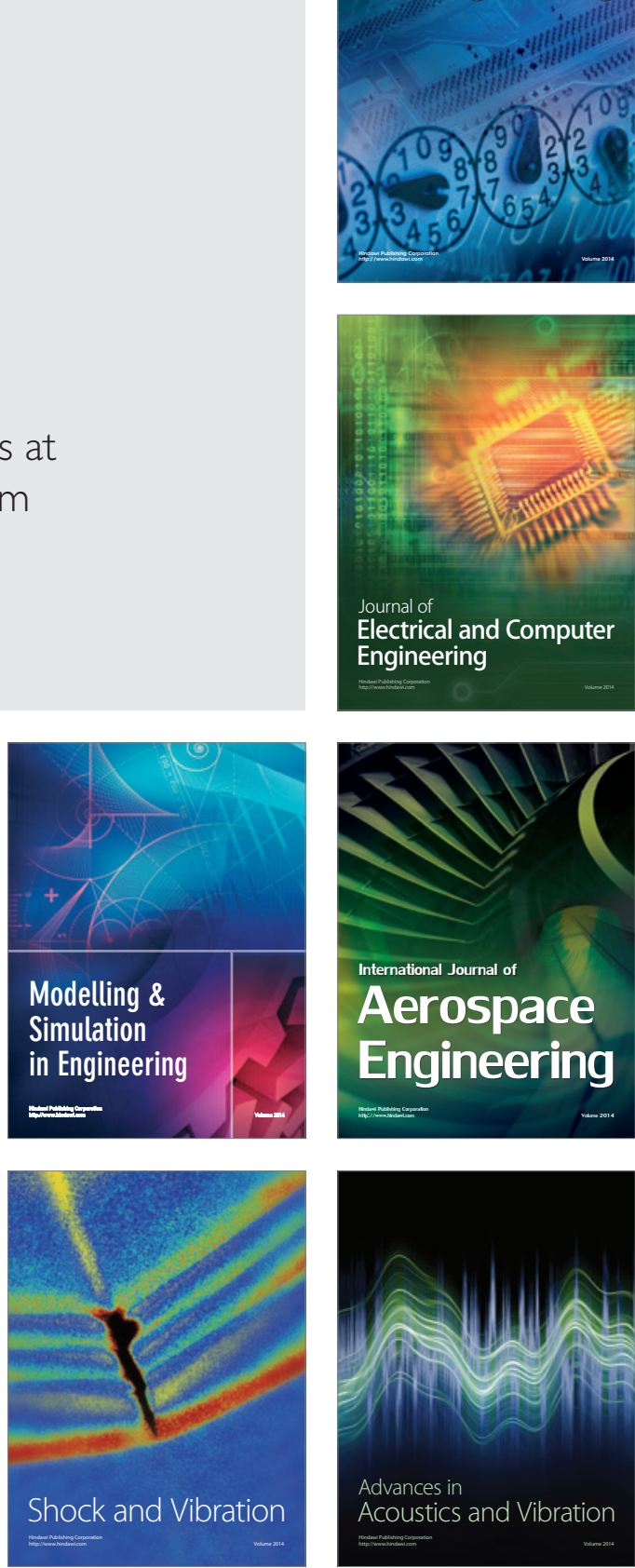